1 Universidade de Brasília (UnB) - Brasília (DF)

Brasil.

patsilveirarodrigues@gmail. com

2 Universidade de Brasília (UnB), Faculdade de Ceilândia (FCE) - Brasília (DF), Brasil.

msodario@yahoo.com.br

3 Universidade de Brasília $($ UnB), Departamento de Farmácia (FAR) - Brasília (DF), Brasil.

nul.tavares@gmail.com

\section{Avaliação da implantação do Eixo Estrutura do Programa Nacional de Qualificação da Assistência Farmacêutica no SUS}

\author{
Evaluation of the implementation of the Axis Structure of the \\ National Pharmaceutical Assistance Qualification Program in the \\ SUS
}

Patrícia Silveira Rodrigues ${ }^{\mathbf{1}}$, Mariana Sodário Cruz², Noemia Urruth Leão Tavares ${ }^{\mathbf{3}}$
RESUMO Neste estudo, analisa-se a implantação do Eixo Estrutura do Programa Nacional de Qualificação da Assistência Farmacêutica em 316 municípios. Foi realizada uma avaliação normativa das dimensões estrutura física/recursos humanos e processo e análise de aspectos do contexto organizacional. Os municípios apresentaram, em sua maioria, grau de implantação insatisfatório (44\%) ou crítico (21\%). Observou-se influência do contexto organizacional no grau de implantação. Destacam-se, também, a cooperação entre os gestores do Sistema Único de Saúde na implantação do Eixo Estrutura e dificuldades na realização do diagnóstico para identificação das necessidades de estruturação da Assistência Farmacêutica.

PALAVRAS-CHAVE Assistência Farmacêutica. Políticas públicas de saúde. Avaliação em saúde. Atenção Primária à Saúde.

ABSTRACT In this study, we analyze the implementation of the Axis Structure of the National Pharmaceutical Assistance Qualification Program in 316 municipalities. It was performed a normative assessment of the physical structure dimensions / human resources and process and analysis of aspects of the organizational context. The municipalities presented, mostly, unsatisfactory (44\%) or critical (21\%) level of implementation. It was noticed some influence of the organizational context on the degree of implementation. Noteworthy are also cooperation between the Unified Health System (SUS) managers in the implementation of the Axis Structure, as well as the difficulties in diagnosis to identify the structuring needs of Pharmaceutical Care.

KEYWORDS Pharmaceutical Services. Health public policies. Health evaluation. Primary Health Care. 


\section{Introdução}

No Brasil, a Constituição Federal de 1988 estabeleceu a saúde como um direito universal que deve ser garantido pelo Estado (BRASIL, 1988). O Sistema Único de Saúde (SUS), institucionalizado pela Lei $\mathrm{n}^{\circ} 8.080 / 90$, possui como princípios doutrinários a universalidade de acesso aos serviços de saúde e a integralidade da assistência, competindo-lhe a execução de ações de assistência terapêutica integral, inclusive farmacêutica (art. $6^{\circ}$ ) (BRASIL, 1990).

$\mathrm{Na}$ perspectiva de concretizar e executar políticas farmacêuticas e com o escopo de promover o acesso a medicamentos essenciais e o uso racional de medicamentos, foram estabelecidas a Política Nacional de Medicamentos (PNM) e a Política Nacional de Assistência Farmacêutica (PNAF) (BRASIL, 2006). Desde então, diversos avanços foram garantidos, dentre estes, destaca-se a ampliação nos investimentos da gestão pública: apenas de recursos federais, o orçamento passou de 1,9 bilhão de reais para 12,4 bilhões, considerando o período de 2003 a 2014 (CONASS, 2014).

Outro avanço a ser considerando é a instituição do Programa Nacional de Qualificação da Assistência Farmacêutica no Sistema Único de Saúde (Qualifar-SUS). O programa Qualifar-SUS é uma estratégia que tem como finalidade contribuir para o processo de aprimoramento e integração das atividades da Assistência Farmacêutica (AF) nas redes de atenção à saúde (BRASIL, 2012B).

Para tanto, o programa está organizado em quatro eixos que se integram entre si, são eles: estrutura, informação, educação e cuidado (BRASIL, 2015B). Os principais objetivos do Qualifar-SUS são a estruturação física dos serviços farmacêuticos, disponibilidade de informações que proporcionem avaliação das ações e serviços de saúde, promoção da educação permanente para o aprimoramento das práticas profissionais e inserção das práticas clínicas no âmbito da AF (BRASIL, 2012B, 2015B).
O primeiro eixo a ser implementado do programa Qualifar-SUS foi o Estrutura, a partir da pactuação das três esferas de gestão do SUS da Portaria MS/GM no 1.215 , de 13 de junho de 2012, que institui apoiar a estruturação da $A F$ na atenção básica dos municípios de até 100 mil habitantes com população em extrema pobreza, constantes do Plano Brasil Sem Miséria (BSM), denominados municípios elegíveis ao Eixo Estrutura (BRASIL, 2012A).

A habilitação dos municípios do Plano BSM com até 100 mil habilitantes, no período de 2012 a 2013, no Eixo Estrutura, ocorreu a partir de seleção nacional com os elegíveis ao programa. A seleção dos municípios priorizou aqueles que participavam de duas iniciativas do Saúde Mais Perto de Você, que é um conjunto de ações do Ministério da Saúde (MS) para reorganização, fortalecimento e estruturação da atenção básica: o Programa Nacional de Melhoria do Acesso e da Qualidade da Atenção Básica (PMAQ-AB); e o Programa de Requalificação das Unidades Básicas de Saúde (Requalifica UBS). Ainda, foi adotado como prioridade na seleção municípios com adesão ao Sistema Hórus ou que possuíssem sistema de informação próprio para a gestão da AF (BRASIL, 2015B).

A implementação do Eixo Estrutura do programa Qualifar-SUS previu, além do apoio técnico aos municípios, a destinação de recursos financeiros de investimento e custeio para reestruturação física e de recursos humanos da AF na atenção básica (BRASIL, 2015B).

Como orientação para implantação do Eixo Estrutura, uma sequência de atividades para implantação do programa é realizada após a habilitação e recebimento dos recursos financeiros pelos municípios habilitados (BRASIL, 2012B, 2015B): (1) Realizar o diagnóstico da situação da $\mathrm{AF}$, principalmente com relação à estrutura física e aos equipamentos; (2) Planejar ações e metas de estruturação baseadas no diagnóstico e realizar, na ferramenta de Controle, Acompanhamento e Avaliação de Resultados (e-CAR) registro do planejamento; (3) Executar as ações e as metas 
planejadas para a estruturação da AF; (4) Enviar o conjunto de dados do Componente Básico de Assistência Farmacêutica para a Base Nacional de Dados de Ações e Serviços da Assistência Farmacêutica do MS; (5) Monitorar o planejamento e a execução das ações de estruturação.

O objetivo desta pesquisa é avaliar o grau de implantação do Eixo Estrutura do Qualifar-SUS nos municípios habilitados, tendo como referência atributos relativos à estrutura física/recursos humanos e ao processo, além de analisar a influência de aspectos relacionados com o contexto político e técnico-organizacional.

\section{Métodos}

Trata-se de um estudo descritivo de caráter avaliativo, que utilizou a avaliação normativa para julgamento da dimensão de estrutura física/recursos humanos e da dimensão processo e incorporou uma pesquisa avaliativa para análise da influência do contexto organizacional que possa ter influenciado o grau de implantação (GI) da intervenção, como proposto por Denis e Champagne (1997).

$O$ alvo desta pesquisa são os municípios habilitados no Eixo Estrutura do
Qualifar-SUS nos anos de 2012 e 2013. Nesse período, 906 municípios foram habilitados no Eixo Estrutura, 453 municípios em cada ano.

Os dados utilizados neste estudo foram coletados a partir de fonte primária (questionário estruturado) e fontes secundárias (análise documental, banco de dados do FormSUS para diagnóstico dos serviços farmacêuticos na atenção básica, banco de dados do Sistema e-CAR e banco de dados da Base Nacional de Dados, Ações e Serviços da Assistência Farmacêutica do MS).

A realização deste estudo ocorreu em cinco etapas: construção do modelo lógico; avaliação da estrutura física/recursos humanos; avaliação do processo; determinação do grau de implantação; e análise de aspectos relacionados ao contexto organizacional.

A primeira etapa do estudo, a construção do modelo lógico do Eixo Estrutura do programa QualifaR-SUS (figura 1), ocorreu por meio da análise dos documentos oficiais do MS relacionados com o programa em questão. Envolveu a identificação dos insumos, atividades, produtos e os resultados em curto e médio prazo que levariam, em longo prazo, ao impacto esperado na $\mathrm{AF}$ básica (CASSIOLATO; GUERESI, 2010). 
Figura 1. Modelo lógico do Eixo Estrutura do programa Qualifar-SUS

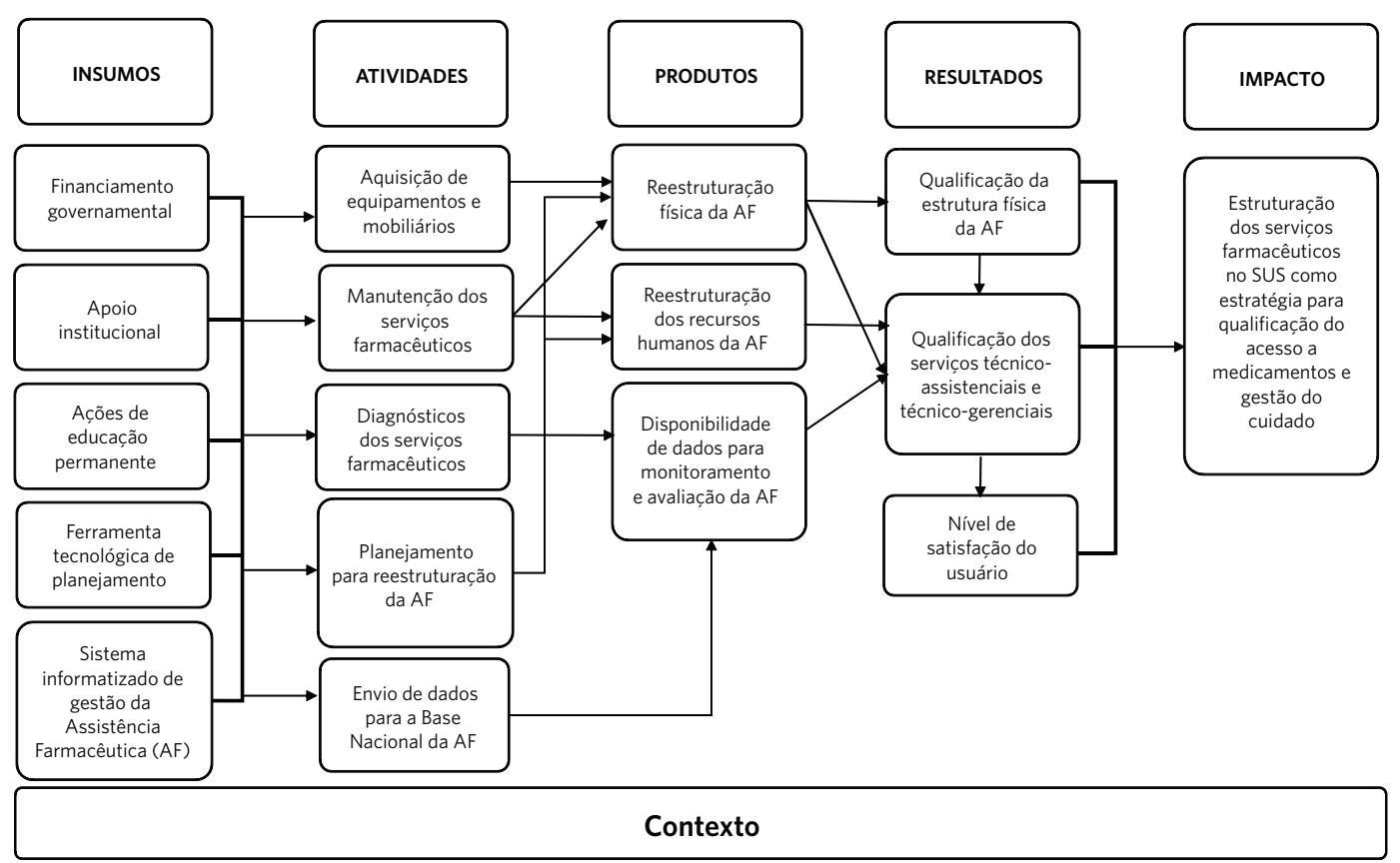

As informações apresentadas no modelo lógico possibilitaram a construção do instrumento estruturado que foi utilizado para determinar o grau de implantação dos municípios habilitados no Eixo Estrutura, por meio do método de avaliação normativa da estrutura física/recursos humanos e do processo, além de investigar aspectos do contexto de implantação.

A coleta de dados foi realizada por intermédio de instrumento eletrônico estruturado e foi enviada aos secretários municipais e ao responsável pelo Eixo Estrutura do programa Qualifar-SUS dos municípios habilitados no período de julho a setembro de 2015 por meio da ferramenta Google Form. As variáveis investigadas para avaliação do grau de implantação foram definidas de acordo com o objetivo de cada dimensão: estrutura física/recursos humanos (existência de mobiliários, equipamentos, conectividade, recursos humanos e estrutura física da Central de Abastecimento Farmacêutico
- CAF) e processo (realização do diagnóstico dos serviços farmacêuticos; planejamento e cadastro das ações e metas no Sistema e-CAR; execução dos recursos financeiros destinados ao Eixo Estrutura; uso de sistema de informação para gestão da AF e envio de dados para a Base Nacional da Assistência Farmacêutica).

Para análise dos dados, foi elaborada uma pontuação (tabela 1), atribuindo-se o mesmo peso às dimensões (10 pontos cada) e às subdimensões ( 2 pontos cada). Os itens que compunham as subdimensões receberam pontuação de acordo com sua relação de importância na estruturação da $\mathrm{AF}$ na atenção básica. Dentro da dimensão estrutura física/ recursos humanos, foi investigada a quantidade suficiente de itens relacionados com a estrutura física aos recursos humanos para o desempenho pleno dos serviços de AF. Já na dimensão processo, levou-se em consideração a realização de cada atividade de implantação do Eixo Estrutura. 
Tabela 1. Pontuação conferida às dimensões e subdimensões para avaliação do grau de implantação nos municípios habilitados no Eixo Estrutura do Qualifar-SUS, 2015

\begin{tabular}{|c|c|c|}
\hline Dimensão & Subdimensão & Pontuação \\
\hline \multirow[t]{5}{*}{ Estrutura física/Recursos humanos (10) } & Mobiliários & 2,0 \\
\hline & Equipamentos & 2,0 \\
\hline & Conectividade & 2,0 \\
\hline & Estrutura física da CAF & 2,0 \\
\hline & Recursos humanos & 2,0 \\
\hline \multirow[t]{5}{*}{ Processo (10) } & $\begin{array}{l}\text { Realização da etapa de diagnóstico para levantamento das } \\
\text { necessidades de estruturação }\end{array}$ & 2,0 \\
\hline & $\begin{array}{l}\text { Planejamento de aç̃̃es e metas para reestruturação da AF } \\
\text { e cadastro no Sistema e-CAR }\end{array}$ & 2,0 \\
\hline & $\begin{array}{l}\text { Execução dos recursos financeiros do Eixo Estrutura do } \\
\text { programa Qualifar-SUS }\end{array}$ & 2,0 \\
\hline & Uso de sistema de informação para gestão da AF & 2,0 \\
\hline & $\begin{array}{l}\text { Envio de dados para a Base Nacional de Dados, Ações e } \\
\text { Serviços da Assistência Farmacêutica }\end{array}$ & 2,0 \\
\hline
\end{tabular}

Fonte: Elaboração própria.

A análise dos dados foi realizada no software Epi-Info 7. Foram utilizados os seguintes parâmetros: intervalo de confiança (IC) entre $97-98 \%$, na frequência esperada (FE) de $50 \%$, limite de confiança (LC) de $5 \%$ e estimativa de amostra mínima de 311 municípios. Como medida de análise, utilizou-se a variável ano de habilitação dos municípios (2012 ou 2013) com os parâmetros de $\mathrm{IC}=85 \%, \mathrm{FE}=50 \%, \mathrm{LC}=5 \%$ e cálculo de amostra mínima de 143 municípios.

Para a definição do grau de implantação, foi utilizado sistema de escores proposto por Felisberto (2001), que permitiu classificar os municípios habilitados no Eixo Estrutura em distintos níveis de implantação: crítico, para pontuações obtidas entre 0,0 a 4,9; insatisfatório de 5,0 a 6,9; satisfatório entre 7,0 a 8,9 e pontuações acima de 9,0 foram consideradas como excelente.

Para análise do contexto organizacional, utilizou-se como fonte de dados o questionário estruturado, documentos relacionados com o Eixo Estrutura do Qualifar-SUS informações do FormSUS de diagnóstico, banco de dados do Sistema e-CAR e também da Base Nacional de Dados, Ações e Serviços da Assistência Farmacêutica.

Como metodologia de análise documental, empregou-se a técnica de análise de conteúdo (BARDIN, 1979). Para tanto, estruturaram-se categorias de análise de acordo com as variáveis contextuais selecionadas: (1) Dimensão político-organizacional (cenário de implantação do Qualifar-SUS, atores envolvidos na formulação do programa e estratégias de implantação) e (2) Dimensão técnico-organizacional (Cooperação dos atores no apoio a implantação, contribuição dos dispositivos institucionais na implantação).

Os dispositivos institucionais, para este estudo, foram identificados como as atividades para implantação do Eixo Estrutura definidas pelo MS, avaliados na dimensão processo.

Os documentos analisados consistiram em: portarias de regulamentação do QualifarSUS, projeto estratégico, manuais e instruções técnicas relacionados ao Eixo Estrutura (BRASIL, 2009, 2012B, 2013A, 2013C, 2014A, 2015). 
A pesquisa foi aprovada pelo Comitê de Ética em Pesquisa (CEP) da Universidade de Brasília (UnB), cujo número do parecer é 1.080.961, e CAAE n ${ }^{\circ} 43176415.5 .0000 .0030$.

\section{Resultados e discussão}

Dos 906 municípios habilitados no Eixo Estrutura no ano de 2012 e 2013, foram recebidas 379 respostas, destas, 316 foram consideradas válidas (participante ser gestor municipal ou responsável pelo programa no município). Quando em respostas concomitantes do gestor municipal e do responsável pelo Eixo Estrutura do mesmo município, foi dada preferência à resposta do responsável, sendo considerada apenas uma resposta do questionário por município.

\section{Caracterização dos municípios parti- cipantes da pesquisa}

Com relação ao total de munícipios participantes da pesquisa e a sua distribuição por região do Brasil, foi observado que: $59,8 \%$ representavam municípios da região Nordeste, $15,1 \%$ da região Sudeste, $10,7 \%$ da região Sul, $10,7 \%$ região Norte e $3,8 \%$ da região CentroOeste. Essa proporção da participação de municípios em cada região brasileira guarda similaridade com a classificação de municípios habilitados no Eixo Estrutura.
Em se tratando da proporção dos municípios participantes em relação ao ano de habilitação, o resultado foi que $45,6 \%$ foram habilitados no ano de 2012 , e $54,4 \%$ correspondiam aos habilitados no ano de 2013.

Quanto ao porte populacional, $77 \%$ são municípios com população menor de 25.000 mil habitantes, $18 \%$ são municípios com população entre 25.001 mil habitantes e 50.000 mil habitantes e $5 \%$ possuem população maior que 50.001 habitantes.

\section{Grau de implantação}

Ao considerar as duas dimensões em conjunto, o grau de implantação do Eixo Estrutura do programa Qualifar-SUS foi considerado como insatisfatório em $44 \%$ dos municípios, satisfatório em $34 \%$, crítico em $21 \%$ e apenas $1 \%$ dos habilitados apresentaram grau de implantação excelente. Desse modo, a estimativa do grau de implantação do Eixo Estrutura em âmbito nacional foi classificada como implantação de grau insatisfatório $(6,5)$ (tabela 2).

Não foi observada variação significativa do grau de implantação entre os municípios habilitados no ano de 2012 (GI=6,7) daqueles habilitados no ano de 2013 (GI=6,3) (tabela 2). As tabelas 3 e 4 sintetizam a pontuação obtida e o grau de implantação correspondente a cada dimensão, subdimensão e item avaliado. Na sequência, serão apresentados e discutidos os resultados de cada dimensão.

Tabela 2. Graus de implantação das dimensões estrutura física/recursos humanos e processo do Eixo Estrutura do programa Qualifar-SUS, 2015

\begin{tabular}{lccr}
\hline Dimensão & Brasil (n=316) & $\begin{array}{c}\text { Habilitados no ano de } \\
\mathbf{2 0 1 2}(\mathbf{n = 1 4 4 )}\end{array}$ & $\begin{array}{c}\text { Habilitados no ano de } \\
\mathbf{2 0 1 3}(\mathbf{n = 1 7 2})\end{array}$ \\
\hline $\begin{array}{l}\text { Estrutura física/Recursos } \\
\text { humanos }\end{array}$ & 5,5 & 5,6 & 5,4 \\
Processo & & & 7,2 \\
Grau de implantação & 7,5 & 7,9 & 6,3 \\
\hline
\end{tabular}

Fonte: Elaboração própria. 


\section{Avaliação da dimensão estrutura físi- $\mathrm{ca} /$ recursos humanos}

Essa dimensão trata da avaliação da suficiência de equipamentos, mobiliários, conectividade, recursos humano e estrutura física da CAF para o funcionamento dos serviços de AF na atenção básica.

O grau de implantação dessa dimensão foi considerado como insatisfatório $(\mathrm{GI}=5,5)$.
Se considerado na avaliação da dimensão o ano de habilitação, não há variação significativa do grau de implantação (tabela 2).

A subdimensão que apresentou melhor desempenho na estrutura física foi a referente a equipamentos $(\mathrm{GI}=6,0)$, seguida da dimensão mobiliário (GI=5,8). Porém, ambas as subdimensões obtiveram grau de implantação insatisfatório (tabela 3).

Tabela 3. Grau de implantação da dimensão estrutura física/recursos humanos do Eixo Estrutura do programa Qualifar-SUS, 2015

\begin{tabular}{|c|c|c|c|c|c|c|}
\hline Dimensão & Subdimensão & Item avaliado & $\begin{array}{l}\text { Pontuação } \\
\text { máxima }\end{array}$ & $\begin{array}{l}\text { Pontuação } \\
\text { obtida }\end{array}$ & Proporção & Grau de implantação \\
\hline \multirow{19}{*}{ 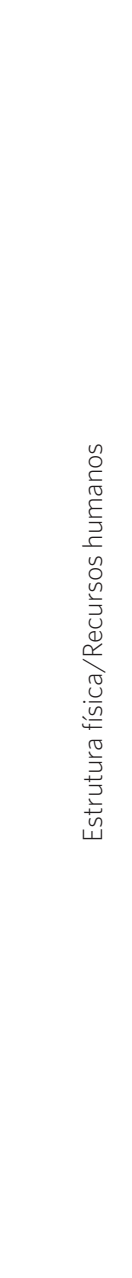 } & \multirow[t]{12}{*}{ Mobiliários } & Mesa para computador & 0,10 & 0,07 & 7,0 & \multirow[t]{12}{*}{ 5,8 (insatisfatório) } \\
\hline & & Cadeira & 0,20 & 0,14 & 7,0 & \\
\hline & & Estantes de aço & 0,20 & 0,13 & 6,5 & \\
\hline & & Armários de aço & 0,20 & 0,12 & 6,0 & \\
\hline & & Balcão com prateleira & 0,20 & 0,11 & 5,5 & \\
\hline & & Mesa com gaveta & 0,10 & 0,07 & 7,0 & \\
\hline & & Mesa auxiliar & 0,10 & 0,06 & 6,0 & \\
\hline & & Caixa tipo BiN & 0,20 & 0,08 & 4,0 & \\
\hline & & Lixeira com tampa e pedal & 0,20 & 0,11 & 5,5 & \\
\hline & & $\begin{array}{l}\text { Bancada revestida de mate- } \\
\text { rial liso e resistente }\end{array}$ & 0,20 & 0,09 & 4,5 & \\
\hline & & $\begin{array}{l}\text { Escada para a retirada de } \\
\text { medicamentos }\end{array}$ & 0,10 & 0,05 & 5,0 & \\
\hline & & Paletes/estrados & 0,20 & 0,12 & 6,0 & \\
\hline & \multirow[t]{5}{*}{ Equipamentos } & Computador & 0,4 & 0,24 & 6,0 & \multirow[t]{5}{*}{ 6,0 (insatisfatório) } \\
\hline & & Impressora & 0,4 & 0,23 & 5,7 & \\
\hline & & $\begin{array}{l}\text { Refrigerador para medica- } \\
\text { mento termossensível }\end{array}$ & 0,4 & 0,26 & 6,5 & \\
\hline & & Ar condicionado & 0,4 & 0,24 & 6,0 & \\
\hline & & Termômetro digital & 0,4 & 0,23 & 5,7 & \\
\hline & \multirow[t]{2}{*}{ Conectividade } & Acesso à internet na CAF & 1 & 0,79 & 7,9 & \multirow[t]{2}{*}{ 5,8 (insatisfatório) } \\
\hline & & $\begin{array}{l}\text { Acesso à internet na(s) } \\
\text { farmácia(s) da atenção } \\
\text { básica }\end{array}$ & 1 & 0,36 & 3,6 & \\
\hline
\end{tabular}




\begin{tabular}{|c|c|c|c|c|c|c|}
\hline \multicolumn{7}{|c|}{ Tabela 1. (cont.) } \\
\hline \multirow{12}{*}{ 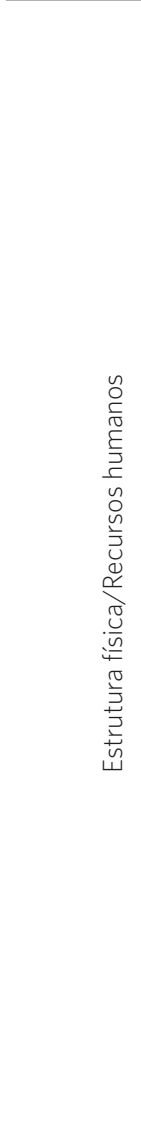 } & $\begin{array}{l}\text { Estrutura física } \\
\text { da CAF }\end{array}$ & $\begin{array}{l}\text { A CAF possui identificação } \\
\text { externa CAF }\end{array}$ & 0,2 & 0,12 & 6,0 & 4,8 (crítico) \\
\hline & & CAF possui piso adequado & 0,2 & 0,17 & 8,5 & \\
\hline & & $\begin{array}{l}\text { A CAF possui paredes } \\
\text { adequadas }\end{array}$ & 0,2 & 0,14 & 7,0 & \\
\hline & & $\begin{array}{l}\text { A CAF possui portas ade- } \\
\text { quadas }\end{array}$ & 0,2 & 0,10 & 5,0 & \\
\hline & & $\begin{array}{l}\text { A CAF possui teto ade- } \\
\text { quado }\end{array}$ & 0,2 & 0,13 & 6,5 & \\
\hline & & $\begin{array}{l}\text { A CAF possui janelas ade- } \\
\text { quadas }\end{array}$ & 0,2 & 0,10 & 5,0 & \\
\hline & & $\begin{array}{l}\text { CAF conta com carrinhos } \\
\text { para movimentação de } \\
\text { mercadorias }\end{array}$ & 0,2 & 0,03 & 1,5 & \\
\hline & & $\begin{array}{l}\text { CAF conta com extintores } \\
\text { de Incêndio Classe B }\end{array}$ & 0,2 & 0,07 & 3,5 & \\
\hline & & $\begin{array}{l}\text { CAF conta com extintores } \\
\text { de Incêndio pó químico }\end{array}$ & 0,2 & 0,06 & 3,0 & \\
\hline & & $\begin{array}{l}\text { CAF conta com extintores } \\
\text { de incêndio carga d'agua }\end{array}$ & 0,2 & 0,05 & 2,5 & \\
\hline & $\begin{array}{l}\text { Recursos huma- } \\
\text { nos }\end{array}$ & Profissional farmacêutico & 1,0 & 0,5 & 5,0 & 5,5 (insatisfatório) \\
\hline & & Atendente de farmácia & 1,0 & 0,6 & 6,0 & \\
\hline
\end{tabular}

Fonte: Elaboração própria.

O MS prevê recursos de investimentos no valor de R\$ 11.200 a R \$ 33.600 no momento da habilitação, dependendo do porte populacional do município, para compra de equipamentos e mobiliários. A partir dos resultados apresentados, levanta-se a hipótese da insuficiência dos recursos financeiros do programa de acordo com a necessidade para a restruturação dos serviços de AF na atenção básica, apontando a necessidade de rediscussão da pactuação de contrapartidas estaduais e municipais na infraestrutura da AF.

Na avaliação da subdimensão conectividade, que apresentou grau insatisfatório de implantação (GI=5,8), o resultado demonstrou que $79 \%$ dos municípios possuíam conectividade na CAF, e apenas $36 \%$ afirmaram ter acesso à internet nas farmácias da atenção básica (tabela 3). A importância desse dado está principalmente no tocante ao uso de sistema de informação para gerenciamento da AF e controle de estoque. O Sistema Hórus, que é ofertado gratuitamente aos municípios pelo MS para gestão da AF, necessita fundamentalmente de conectividade para uso, já que é disponibilizado em plataforma on-line.

As estruturas físicas das CAFs mostraram-se inadequadas para a guarda de medicamentos e insumos para a saúde $(\mathrm{GI}=4,8)$, de acordo com o manual de diretrizes para a estruturação de farmácias no SUS (BRASIL, 2009). Entre os itens dessa subdimensão, a menor nota foi inferida para os equipamentos de controle a incêndio. Em média, 
apenas $30 \%$ dos estabelecimentos contavam com extintores, risco importante quando grande parte das CAFs e farmácias da atenção básica funcionam em casas de alvenarias adaptadas. A precariedade estendeu-se para aspectos das instalações prediais, como teto, piso e parede não adequados, indicando ausência de condições sanitárias para o armazenamento de medicamentos.

Ressalta-se que nenhum dos programas do governo federal para qualificação de infraestrutura prevê recursos financeiros para construção, reforma ou ampliação predial da CAF ou almoxarifado dos estados ou municípios. O financiamento previsto no Requalifica UBS possibilita apenas investimentos nas farmácias das UBS.

Na determinação do grau de implantação para a subdimensão recursos humanos da $\mathrm{AF}$, o total de $54 \%$ dos municípios não tinha farmacêuticos em números suficientes para a manutenção dos serviços. Em contraponto, $40 \%$ dos participantes da pesquisa indicaram necessidade de auxiliares de farmácia.

Os estudos de Canabarro e Hahn (2009), De Bernardi et al. (2006), Silva Júnior e Nunes (2012), Mendes et al. (2014) e Oliveira et al. (2011) também apresentam dados da precariedade dos elementos estruturais da AF, como espaços físicos ineptos, infraestrutura de equipamentos e mobiliários insatisfatória e até mesmo a insuficiência e sobrecarga de profissionais, corroborando os achados desta pesquisa.

\section{Avaliação da dimensão processo}

Em relação à dimensão processo, foram avaliadas as seguintes subdimensões: diagnóstico dos serviços farmacêuticos; planejamento e cadastro das ações e metas no Sistema e-CAR; execução dos recursos financeiros destinados ao Eixo Estrutura; uso de sistema de informação para gestão da $\mathrm{AF}$ e envio de dados para a Base Nacional da Assistência Farmacêutica (tabela 4).

A dimensão processo foi classificada com grau de implantação satisfatório (GI= 7,5). Não houve variação na avaliação se considerado o ano de habilitação dos municípios (tabela 2).

A subdimensão diagnóstico dos serviços farmacêuticos foi classificada com o grau crítico de implantação, com apenas 35\% dos municípios realizando esta ação (tabela 4).

Tabela 4. Grau de implantação da dimensão processo do Eixo Estrutura do programa Qualifar-SUS, 2015

\begin{tabular}{|c|c|c|c|c|c|c|}
\hline Dimensão & Subdimensão & Item avaliado & $\begin{array}{l}\text { Pontuação } \\
\text { máxima }\end{array}$ & $\begin{array}{l}\text { Pontuação } \\
\text { obtida }\end{array}$ & Proporção & Grau de implantação \\
\hline \multirow[t]{5}{*}{ Processo } & $\begin{array}{l}\text { Diagnóstico dos serviços } \\
\text { farmacêuticos }\end{array}$ & $\begin{array}{l}\text { Registro do diagnóstico no } \\
\text { FormSUS disponibilizado }\end{array}$ & 2,0 & 0,71 & 3,5 & 3,5 (crítico) \\
\hline & $\begin{array}{l}\text { As ações e metas plane- } \\
\text { jadas e cadastradas no } \\
\text { Sistema e-CAR }\end{array}$ & $\begin{array}{l}\text { Registro do planejamento } \\
\text { no e-CAR }\end{array}$ & 2,0 & 1,88 & 9,4 & 9,4 (excelente) \\
\hline & $\begin{array}{l}\text { Execução dos recursos } \\
\text { financeiros do Eixo Es- } \\
\text { trutura }\end{array}$ & $\begin{array}{l}\text { Execução dos recursos } \\
\text { financeiros }\end{array}$ & 2,0 & 1,56 & 7,8 & 7,8 (satisfatório) \\
\hline & $\begin{array}{l}\text { Uso de sistema de infor- } \\
\text { mação para gestão da AF }\end{array}$ & $\begin{array}{l}\text { Uso de sistema de infor- } \\
\text { mação }\end{array}$ & 2,0 & 1,73 & 8,6 & 8,6 (satisfatório) \\
\hline & $\begin{array}{l}\text { Envio de dados para a } \\
\text { Base Nacional da Assis- } \\
\text { tência Farmacêutica }\end{array}$ & Envio de dados & 2,0 & 1,60 & 8,0 & 8,0 (satisfatório) \\
\hline
\end{tabular}

Fonte: Elaboração própria. 
Os dados para avaliação da subdimensão diagnóstico foram obtidos na base de dados do formulário on-line FormSUS, que é de administração do MS. O formulário é disponibilizado pela gestão federal do SUS em período definido, que compreende os seis primeiros meses de habilitação no programa, e tem o objetivo de levantar as necessidades de estruturação nas CAF e farmácias da atenção básica.

No entanto, apesar do dado crítico apresentado na execução da etapa de diagnóstico, a etapa de planejamento das ações e metas para estruturação da $\mathrm{AF}$ foi realizada por 94\% dos municípios participantes da pesquisa, sendo avaliada com o grau excelente de implantação (tabela 4).

O diagnóstico para identificação das necessidades de estruturação deveria anteceder a atividade de planejamento, entretanto, não foi o identificado. Contudo, é necessário considerar que o cadastro das ações e metas no sistema e-CAR, também de administração do MS, é condição para o pagamento de recurso financeiro de custeio aos municípios habilitados. O mesmo não ocorre com a realização da atividade de diagnóstico.

A avaliação da subdimensão execução dos recursos financeiros foi avaliada como satisfatória $(\mathrm{GI}=7,8), 65 \%$ dos municípios referiram execução total dos recursos financeiros, $26 \%$ execução parcial e $8 \%$ não executaram os recursos financeiros (tabela 4).

Análise dos municípios que indicaram dificuldades no uso do recurso de investimento repassados pela união $(n=129), 78 \%$ relataram impasses com o processo licitatório. Com relação aos que apontaram problemas com a execução do recurso de custeio, $54 \%(n=114)$ alegaram a mesma dificuldade.

Documento recente publicado pelo Conselho Nacional de Secretários de Saúde (Conass) indica as dificuldades que estados e municípios, principalmente os pequenos municípios, possuem de dificuldades em relação à estrutura burocrática da lei que rege as compras públicas. As gestões públicas enfrentam problemas, como licitações desertas, preços abusivos e descumprimento dos prazos de entrega pelos fornecedores, gerando transtornos de grande impacto ocasionado pelo desabastecimento da rede (BRASIL, 2014B).

Uma das condições para o repasse de recursos de custeio aos municípios habilitados é o envio de dados do componente básico da AF para a Base Nacional de Dados de Ações e serviços da Assistência Farmacêutica do MS, por meio do uso de sistema de informação de gestão da AF. Para tanto, é realizado monitoramento dos municípios com frequência trimestral para repasse do pagamento. $\mathrm{Na}$ avaliação da subdimensão envio de dados para a Base Nacional da Assistência Farmacêutica, considerou-se o envio de informações no intervalo de 4 monitoramentos após o início da avaliação pela gestão Qualifar-SUS. Dessa forma, se os municípios receberam, no período citado, alguma parcela dos recursos financeiros, foi considerada a nota máxima para o item.

Sendo assim, 80\% dos participantes da pesquisa apresentaram envio de dados à Base Nacional de Dados de Ações e serviços da Assistência Farmacêutica. No entanto, 86 $\%$ dos municípios referiram utilizar algum tipo de sistema de informação para gestão da AF. Foi alcançado o grau de implantação satisfatório para ambas as subdimensões.

\section{Análise do contexto}

A análise do contexto de implantação do Eixo Estrutura está apresentada a partir de duas dimensões: político-organizacional e técnico-organizacional.

$\mathrm{Na}$ dimensão político-organizacional, foi possível observar que a elaboração do Qualifar-SUS surge como resposta de intervenção para mudança do cenário deficitário e fragmentado apresentado na $\mathrm{AF}$ dos estados e municípios, evidenciado em auditorias dos órgãos Tribunal de Contas da União (TCU) e Controladoria Geral da União (CGU). 
As constatações apresentadas nos relatórios operacionais de auditorias resultaram na publicação do acordão do TCU n ${ }^{\circ} 1.459 / 2011$, que determinou ao MS definição, no período de 120 dias, do rol de informações mínimas sobre a gestão da AF básica a serem encaminhadas obrigatoriamente pelos estados e municípios que não tivessem aderido ao Sistema Hórus, além do fluxo e cronograma do envio dessas informações. No citado documento, também é recomendada a gestão federal indução de incentivo financeiro para promover fomento ao uso do Sistema Hórus, assim como o desenvolvimento de tecnologias para interoperabilidade de sistemas que garantissem o envio das informações da AF dos municípios que utilizassem sistemas de gestão diferente do Hórus, a União (BRASIL, 2011).

Como encaminhamento as deliberações prolatadas no acordão, é realizada proposta do programa Qualifar-SUS, instituído com quatro diferentes eixos, entre eles, o Eixo Estrutura.

A pactuação do programa Qualifar-SUS foi acompanhada de um intenso processo de articulação entre atores da gestão da AF do MS, outros atores de áreas afins da gestão federal e representações políticas dos estados (Conass) e dos municípios (Conselho Nacional de Secretarias Municipais de Saúde - Conasems).

Notou-se importante cooperação entre os atores envolvidos na implantação, principalmente com o Conass e Conasems, o que possibilitou e favoreceu o desenvolvimento e pactuação do marco normativo que instituiu o Qualifar-SUS e o Eixo Estrutura do programa.

Com relação às estratégias de implantação do Eixo Estrutura do Qualifar-SUS, foi possível identificar fatores que contribuíram de forma sinérgica ou antagônica ao desenvolvimento do programa.

A definição em destinar os investimentos iniciais do Eixo Estrutura aos municípios do Plano BSM afirmou-se com importância positiva, já que o referido Plano foi uma estratégia prioritária do governo federal e teve o objetivo de superar a extrema pobreza até o final do ano de 2014. Para tanto, foi desenvolvida agenda positiva em todas as áreas de políticas públicas, com prioridade para as políticas públicas de saúde e preferência na destinação orçamentária para execução dos objetivos propostos (JAIME; AMARAL; MAGALHÃES JUNIOR, 2014).

Não obstante, a preferência na destinação das ações do Eixo Estrutura para os municípios contemplados com o Plano BSM promoveu dissensos na pactuação do Eixo Estrutura, uma vez que a precariedade da infraestrutura da AF é observada em âmbito nacional como constatado em diversos estudos e na produção dos órgãos de controle brasileiros (BRASIL, 2011; VIEIRA, 2010; VIEIRA; ZUCCHI, 2013).

Com relação à dimensão técnico-organizacional, foi possível analisar o envolvimento dos atores no processo de implantação do Eixo Estrutura. Resultado da pesquisa com os municípios habilitados demonstrou que $72 \%$ dos municípios sinalizaram receber apoio do MS, destes, $41 \%$ referem ter recebido ajuda por meio do apoio institucional da gestão federal do SUS. 21\% relataram receber apoio da Secretária Estadual de Saúde, e 14\% foi o resultado dos que referiram receber apoio dos Conselhos de Secretarias Municipais de Saúde (Cosems). O mesmo resultado de 14\% foi observado nos municípios que relataram não ter recebido nenhum apoio.

Os resultados do apoio à implantação do Eixo Estrutura indicam pouco envolvimento das gestões estaduais com os municípios habilitados. Esse dado rompe com o texto constitucional do pacto federativo (BRASIL, 1988), que direciona o processo de implementação das políticas públicas mediante o princípio da descentralização e relação solidária entre os entes. Os gestores estaduais possuem importante papel de coordenar o processo de implantação das políticas de saúde no SUS do respectivo estado, a partir do apoio técnico e financeiro aos municípios. Todavia, 
é possível avaliar positivamente a interface entre o MS no apoio aos municípios, principalmente em relação à interação da figura do apoio institucional com os habilitados.

$\mathrm{Na}$ análise da contribuição dos dispositivos institucionais na implantação do Eixo Estrutura, foi possível observar que a realização das ações do diagnóstico, etapa de pior desempenho nas atividades de implantação do Eixo Estrutura, é identificada com fragilidades e como uma etapa burocrática, pensada a partir das necessidades da gestão federal.

Reforçam os dados descritos a dificuldade dos municípios no entendimento do objetivo em realizar o diagnóstico para identificação as necessidades de estruturação da AF. Com relação aos que referiram não ter realizado o diagnóstico $(n=44), 64 \%$ expressaram a dificuldade em compreender como executar essa etapa, $18 \%$ desconhecimento total da atividade e os demais $18 \%$ não souberam informar.

Fatores evidenciados no contexto da implantação de um programa podem influenciar os efeitos da intervenção. Essa afirmação justifica a importância do uso do método de análise do contexto na compreensão da determinação do grau de implantação de uma intervenção. Esse fundamento é necessário ao analisar a dificuldade dos municípios em realizar o diagnóstico proposto pela gestão federal do programa, já que é possível levantar a hipótese da interferência do contexto da formulação do programa nos resultados da implantação do Eixo Estrutura.

A gestão federal do Qualifar-SUS tem objetivo próprio na execução da atividade do diagnóstico pelos municípios, que se justifica no interesse de levantamento de informações. Contudo, o propósito principal para os municípios em obter dados se desenvolve no sentido de reconhecer a realidade da AF municipal para traçar estratégias e prioridades locais a partir do financiamento recebido.

Situação similar acontece na análise da atividade de planejamento e cadastro no
Sistema e-CAR das ações e metas para estruturação da AF. Pontos importantes foram sinalizados pelos municípios, como a dificuldade de compreensão da importância em realizar a atividade de planejamento a partir da habilitação no Eixo Estrutura e dificuldades em operar o Sistema e-CAR.

Apesar das dificuldades apontadas na etapa de planejamento e registro no e-CAR, este componente foi avaliado com grau de implantação excelente. Entretanto, esclarece-se que, para análise da implantação dessa etapa, apenas foi verificado o registro do planejamento no sistema, não sendo avaliada a qualidade do conteúdo, assim como não foi verificado o monitoramento das ações e metas cadastradas, ação fundamental para o processo de planejamento.

Ainda na etapa de planejamento, foi possível determinar nos municípios habilitados que apenas em $66 \%$ (n=256) o secretário municipal de saúde participou do planejamento de ações e metas para implantação do programa, em $74 \%$ dos municípios, houve a participação do coordenador municipal de $\mathrm{AF}$ ou farmacêutico e apenas em $17 \%$ incluiu a presença de membro ligado ao planejamento da Secretaria Municipal de Saúde. Em 38\% $(n=256)$ dos municípios, o planejamento foi realizado apenas por um profissional, sem a participação de nenhum outro membro da equipe, em $48 \%$ desses municípios, esse profissional era o coordenador da $\mathrm{AF}$ ou farmacêutico. Esses dados refletem que o planejamento é conduzido de modo pouco interativo entre equipe saúde e gestores, reafirmado a suposição de planejamento não ascendente.

Por fim, foi realizada a análise das subdimensões execução de recursos financeiros, uso do sistema de informação e envio de dados conjuntamente, por guardarem grande interface entre si.

A gestão federal do Eixo Estrutura aponta dificuldades na execução financeira e continuidade do recebimento desses recursos relacionados com o uso de sistema de 
informação da AF pelos municípios habilitados no programa, ante as dificuldades de infraestrutura de conectividade, formação de recursos humanos e demais problemas técnicos.

A pesquisa realizada com os municípios revelou que $88 \%(n=273)$ dos municípios que utilizavam sistema de informação da AF faziam uso do Sistema Hórus, que por ser um sistema $w e b$, tem a necessidade de $500 \mathrm{kbps}$ de conexão à internet para cada subestação de trabalho de distribuição e/ou dispensação de medicamentos (BRASIL, 2014B). Todavia, a dificuldade com conectividade foi relatada em $54 \%(n=239)$ dos municípios que utilizavam o Hórus como sistema de informação.

Dados da Agência Nacional de Telecomunicações (Anatel) demonstram a carência de infraestrutura de acesso à internet, principalmente nos municípios de pequeno porte no interior do País (BRASIL, 2014C), sendo este o principal perfil dos municípios habilitados no Eixo Estrutura. A partir do exposto, o contexto da infraestrutura de conectividade dos pequenos municípios brasileiros e a forma de operacionalização do Sistema Hórus podem ser considerados como um ponto negativo na implantação do Eixo Estrutura.

Por outro lado, é avaliado positivamente o desenvolvimento de tecnologias como o WebService para atender o envio de dados para aqueles municípios que utilizam outros sistemas que não o Hórus para gestão da AF.

Outro ponto levantado está relacionado com o cronograma de monitoramento do envio de dados da AF do município ao MS. Como a legislação que regulamenta o Eixo Estrutura prevê, é condição para o recebimento dos recursos de custeio realização de monitoramento trimestral para avaliação do conjunto de dados enviado para a Base Nacional da Assistência Farmacêutica do MS (BRASIL, 2012). A ausência de envio de dados em um único mês que compõe o trimestre indica suspensão dos recursos, o que pode vir a comprometer a execução das ações planejadas para implantação do programa.

Ao analisar o contexto de implantação do Eixo Estrutura do Qualifar-SUS, é possível supor que o modelo de implementação desse programa é do tipo tradicional ou também chamado top-down (de cima para baixo). Essa evidência surge a partir da constatação de que o poder de decisão é prioritariamente da gestão federal do Eixo Estrutura, os demais entes são vistos como meros implementadores.

A caracterização da verticalidade da gestão do Eixo Estrutura pôde ser observada, principalmente, na definição das atividades de implantação, construção dos instrumentos a serem utilizados e até mesmo na determinação de responsabilidades entre os atores envolvidos na implantação. Essa afirmação se reforça com o pouco (ou irrisório) diagnóstico que é realizado pelos municípios e também na execução mecânica de algumas atividades de implantação, com o objetivo apenas de garantir o recebimento dos recursos financeiros, sem reconhecer a real importância da sua realização para mudanças na $\mathrm{AF}$.

\section{Considerações finais}

Os resultados obtidos neste estudo inédito indicaram que a implantação do Eixo Estrutura do programa Qualifar-SUS alcançou grau de implantação satisfatório em somente um terço dos municípios. Em mais da metade dos habilitados, o grau de implantação foi considerado como insatisfatório ou crítico.

Foi possível observar que a precariedade e insuficiência na estrutura de equipamentos, mobiliários, conectividade, estrutura física das CAFs e recursos humanos da $\mathrm{AF}$ contribuíram para o grau de implantação insatisfatório da dimensão estrutura física/ recursos humanos em grande parcela dos municípios habilitados, aproximando-se, em alguns dos itens analisados, do grau crítico de implantação. 
No tocante à avaliação da implantação da dimensão processo, apesar do positivo resultado em algumas subdimensões, identificaram-se fragilidades principalmente nas etapas diagnóstico e execução dos recursos financeiros, avaliadas com o grau crítico e insatisfatório respectivamente. A partir dos resultados apresentados, sugere-se rediscussão e redimensionamento do processo de diagnóstico e planejamento da gestão da $\mathrm{AF}$ como uma atividade interativa que influencie o processo circunstancial na tomada de decisão para reestruturação da AF municipal, extinguindo a execução desconectada e fragmentada apenas para recebimento dos recursos financeiros.

Entre os fatores antagônicos levantados neste estudo, reconhece-se a precariedade histórica de infraestrutura nos serviços de saúde da atenção básica, como dificuldades no acesso a conectividade e problemas administrativos da gestão em saúde (como os obstáculos regionais enfrentados na compra de bens e serviços ou contratação de recursos humanos). Contudo, problemas como os citados não serão resolvidos apenas com a implantação do Eixo Estrutura, sendo necessária uma ação de cunho nacional e intersetorial.

É fundamental considerar que este estudo se propôs a realizar uma avaliação inicial dos resultados de uma política em franco processo de implantação. Dessa forma, os resultados evidenciados poderão ser utilizados para o aperfeiçoamento do programa Qualifar-SUS e para os importantes avanços propostos por essa estratégia.

Identifica-se como limitação deste estudo a adoção de escores similares para a avaliação das dimensões estrutura e processo. Entretanto, apesar do relatado, como não houve discrepâncias entre as notas obtidas utilizando a escala definida, foi tomada como opção metodológica de atribuir os mesmos escores entre as dimensões e subdimensões escolhidas.

Aponta-se como sugestão para estudos futuros revisão dos critérios de avaliação adotados neste estudo, a partir de metodologias de consenso entre especialistas da área de AF. Igualmente, propõe-se o desenvolvimento de análises regionais que promovam um aprofundamento nas questões levantadas, já que a amostra de municípios obtida não era representativa para tal fim.

Análises do contexto organizacional no âmbito político e técnico em que o programa foi desenvolvido demonstraram correlações sinérgicas e antagônicas que influenciam a implementação do Eixo Estrutura. Fatores positivos, como o alinhamento político entre gestores federais do programa Qualifar-SUS, o Conasems e o Conass na pactuação do programa; a priorização dos municípios do Plano BSM para iniciar a implantação do Eixo Estrutura; e a participação ativa dos gestores das esferas municipal e federal na implantação, apesar da menor mobilização e envolvimentos dos gestores estaduais, sinalizam cenário positivo de cooperação para uma efetiva consolidação do Eixo Estrutura. 


\section{Referências}

BARDIN, Laurence. Análise de conteúdo. Lisboa: edições 70, 1979.

BRASIL. Constituição da República Federativa do Brasil de 1988. Nós, representantes do povo brasileiro, reunidos em Assembleia Nacional Constituinte para instituir um Estado Democrático, destinado a assegurar o exercício dos direitos sociais e individuais, a liberdade, a segurança, o bem-estar, o desenvolvimento, a igualdade e a justiça como valores supremos de uma sociedade fraterna, pluralista e sem preconceitos, fundada na harmonia social e comprometida, na ordem interna e internacional, com a solução pacífica das controvérsias, promulgamos, sob a proteção de Deus, a seguinte CONSTITUIÇÃO DA REPÚBLICA FEDERATIVA DO BRASIL. Diário Oficial [da] União. Brasília, DF, 05 de outubro de 1988. Disponível em: <http://www.planalto.gov.br/ccivil_03/constituicao/ constituicaocompilado.htm>. Acesso em: 12 nov. 2016.

Lei ${ }^{\circ}$ 8.080, de 19 de setembro de 1990. Dispõe sobre as condições para a promoção, proteção e recuperação da saúde, a organização e o funcionamento dos serviços correspondentes e dá outras providências. Diário Oficial [da] União. Brasília, DF, 20 de setembro de 1990. Disponível em: <http://www.planalto.gov.br/ ccivil_03/leis/L8080.htm>. Acesso em: 12 nov. 2016.

Ministério da Saúde. Portaria ${ }^{\circ}$ 1. 215, de 13 de junho de 2012. Regulamenta a transferência de recursos destinados ao Eixo Estrutura do Programa Nacional de Qualificação da Assistência Farmacêutica (Qualifar-SUS) no âmbito do Sistema Único de Saúde (SUS). Diário Oficial [da]União. Brasília, DF, 14 de junho de 2012. Disponível em: <http://www.lex.com. br/legis_23450520_PORTARIA_N_1215_DE_13_DE_ JUNHO_DE_2012.aspx>. Acesso em: 12 nov. 2016.

Ministério da Saúde. Secretaria de Ciência, Tecnologia e Insumos Estratégicos. Departamento de Assistência Farmacêutica Insumos Estratégicos. Assistência farmacêutica na atenção básica: instruções técnicas para sua organização. 2. ed. Brasília, DF: Ministério da Saúde, 2006. Disponível em: <http:// www.ensp.fiocruz.br/portal-ensp/judicializacao/ pdfs/283.pdf>. Acesso em: 12 nov. 2016.

Ministério da Saúde. Secretaria de Ciência, Tecnologia e Insumos Estratégicos. Departamento de Assistência Farmacêutica Insumos Estratégicos. Cuidado farmacêutico na atenção básica. Brasília, DF: Ministério da Saúde, 2014a.

Ministério da Saúde. Secretaria de Ciência, Tecnologia e Insumos Estratégicos. Departamento de Assistência Farmacêutica Insumos Estratégicos. Diretrizes para estruturação de farmácias no âmbito do Sistema Único de Saúde. Brasília, DF: Ministério da Saúde, 2009 (Série A. Normas e Manuais Técnicos). Disponível em: $<$ http://www. cff.org.br/userfiles/40\%20-\%20BRASIL_\%20 MINIST\%C3\%89RIO\%20DA\%20SA\%C3\%9ADE\%20 2009\%20Diretrizes\%20para\%20Estruturacao\%20 Farmacias\%20no\%20Ambito\%20do\%20SUS.pdf > . Acesso em: 12 nov. 2016.

Ministério da Saúde. Secretaria de Ciência, Tecnologia e Insumos Estratégicos. Departamento de Assistência Farmacêutica Insumos Estratégicos. Documento Técnico do Projeto Estratégico Eixo Estrutura - QUALIFAR-SUS: apoio a estruturação dos serviços farmacêuticos na atenção básica dos municípios do Plano Brasil Sem Miséria. Brasília, DF Ministério da Saúde, 2013a.

Ministério da Saúde. Secretaria de Ciência, Tecnologia e Insumos Estratégicos. Departamento de Assistência Farmacêutica Insumos Estratégicos. Hórus - Sistema Nacional de Gestão da Assistência Farmacêutica. Brasília, DF: Ministério da Saúde, 2014b. Disponível em: <http://portalsaude.saude.gov.br/index. php/o-ministerio/principal/secretarias/sctie/horus>. Acesso em: 12 nov. 2016.

Ministério da Saúde. Secretaria de Ciência, Tecnologia e Insumos Estratégicos. Departamento de Assistência Farmacêutica Insumos Estratégicos. Relatório oficinas nacionais Eixo Estrutura programa QUALIFAR-SUS ano 2013. Brasília, DF: Ministério da Saúde, 2013c. 
Ministério da Saúde. Secretaria de Ciência, Tecnologia e Insumos Estratégicos. Departamento de Assistência Farmacêutica Insumos Estratégicos. QUALIFAR-SUS - Eixo Estrutura: orientações técnicas. Brasília, DF: Ministério da Saúde, 2012b. Disponível em: <http://u.saude.gov.br/images/pdf/2014/abril/03/ manual-instrutivo-qualifarsus-283-29.pdf $>$. Acesso em: 12 nov. 2016.

Ministério da Saúde. Secretaria de Ciência, Tecnologia e Insumos Estratégicos. Departamento de Assistência Farmacêutica Insumos Estratégicos. QUALIFAR-SUS: eixo estrutura - atenção básica instruções técnicas. Brasília, DF: Ministério da Saúde, 2015. Disponível em: <http://portalsaude. saude.gov.br/index.php/o-ministerio/principal/ leia-mais-o-ministerio/216-sctie-raiz/daf-raiz/ ceaf-sctie/qualifarsus-raiz/eixo-estrutura/11-eixoestrutura/20645-eixo-estrutura>. Acesso em: 12 nov. 2016.

Ministério das Telecomunicações. Agência Nacional de Telecomunicações. Qualidade da Banda Larga Fixa (SCM): relatório de indicadores de desempenho operacional. Brasília, DF: Ministério da Saúde, 2014c. Disponível em: <http://www.anatel.gov. br/Portal/verificaDocumentos/documento.asp?numer oPublicacao $=331437 \&$ filtro $=1 \&$ documentoPath $=331437$. pdf $>$. Acesso em: 7 jan. 2016.

. Tribunal de Contas da União. Promoção da assistência farmacêutica e insumos estratégicos na atenção básica em saúde: relatório de auditoria operacional. Brasília, DF: TCU, 2011.

CANABARRO, I. M.; HAHN, S. Panorama da assistência farmacêutica na saúde da família em município do interior do estado do Rio Grande do Sul. Epidemiol. Serv. de Saúde, Brasília, DF, v. 18, n. 4, p. 345355, out./dez. 2009.

CASSIOLATO, M.; GUERESI, S. Como elaborar Modelo Lógico: roteiro para formular programas e organizar avaliação. Brasília, DF: IPEA, 2010. Disponível em: $<$ http://www.ipea.gov.br/portal/images/stories/ PDFs/100924_notatec6disoc.pdf>. Acesso em: 12 out. 2016.

\section{CONSELHO NACIONAL DE SECRETÁRIOS} DE SAÚDE (CONASS). Nota técnica $n^{\circ} 15 / 2014$. Apresentação das dificuldades enfrentadas pelas Secretarias Estaduais de Saúde na aquisição de medicamentos. Brasília, DF: CONASS, 2014.

DE BERNARDI, C. L. B.; BIEBERBACH, E. W.; THOMÉ, H. I. Avaliação da assistência farmacêutica básica nos municípios de abrangência da 17a Coordenadoria Regional de Saúde do Rio Grande do Sul. Saúde soc., São Paulo, v. 15, n. 1, p. 73-83, jan./abr. 2006.

DENIS, J. L.; CHAMPAGNE, F. Análise da Implantação. In: HARTZ, Z. M. A. (Org.). Avaliação em Saúde: dos modelos conceituais à prática na análise de implantação de programas. Rio de Janeiro: Fiocruz, 1997, p. 49-88.

FELISBERTO, E. Avaliação do processo de implantação da estratégia da atenção integrada às doenças prevalentes da infância (AIDPI) no programa saúde da família (PSF) no Estado de Pernambuco no período de 1998 a 1999. 2001. 78 f. Dissertação (Mestrado em Saúde Pública) - Centro de Pesquisas Aggeu Magalhães, Fundação Oswaldo Cruz, Recife, 2001.

\section{JAIME, P. C.; AMARAL, M. A.; MAGALHÃES}

JUNIOR, H. M. Ações de saúde no Plano Brasil Sem Miséria: o acesso à saúde orientado pela superação de iniquidades. In: CAMPELLO, T., FALCÃO, T., COSTA, P. V. (Org.). O Brasil Sem Miséria. Brasília, DF: Ministério da Saúde, 2014. p. 603-631.

MENDES, L. V. et al. Disponibilidade de medicamentos nas unidades básicas de saúde e fatores relacionados: uma abordagem transversal. Saúde debate, Rio de Janeiro, v. 38, n. esp., p. 109-123, out. 2014.

OLIVEIRA, L. C. F.; ASSIS, M. M. A.; BARBONI, A. R. Avaliação da assistência farmacêutica básica em um município da Bahia, Brasil. Rev. baiana saúde pública, Salvador, v. 34, n. 4, p. 853-864, out./dez. 2011.

SILVA JÚNIOR, E. B.; NUNES, L. M. N. Avaliação da Assistência Farmacêutica na atenção primária no município de Petrolina (PE). Arquivos Brasileiros de 
Ciências da Saúde, São Paulo, v. 37, n. 2, p. 65-69, maio/ ago. 2012. Disponível em: <http://bases.bireme.br/cgibin/wxislind.exe/iah/online/?IsisScript=iah/iah.xis\& $\mathrm{src}=$ google \&base $=$ LILACS\&lang $=\mathrm{p} \&$ nextAction $=\operatorname{lnk} \&$ exprSearch=646084\&indexSearch=ID>. Acesso em: 6 mar. 2016.

VIEIRA, F. S. Farmacêutica no sistema público de saúde no Brasil. Rev. Panam. Salud Publica, Washington, v. 27, n. 2, p. 149-156, fev. 2010.
VIEIRA, F. S.; ZUCCHI, P. Financiamento da assistência farmacêutica no sistema único de saúde. Saúde soc., São Paulo, v. 22, n. 1, p. 73-84, jan./mar. 2013.

Recebido para publicação em março de 2016

Versão final em setembro de 2016

Conflito de interesses: inexistente

Suporte financeiro: não houve 